Hautarzt 2020 · 71:917-918

https://doi.org/10.1007/s00105-020-04722-z

Online publiziert: 22. Oktober 2020

(c) Springer Medizin Verlag GmbH, ein Teil von Springer Nature 2020
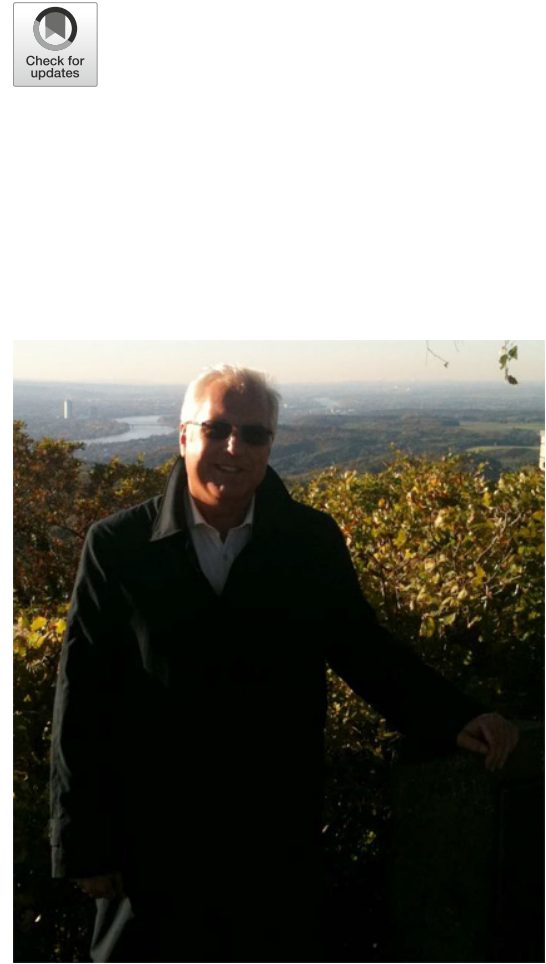

Abb. 1 ॥ Prof. Dr. Alexander Kapp. (@ privat)

Das Jahr 2020 ist sicher eines, das alle, die es erlebt haben, nicht vergessen werden. Was diese Zeitschrift betrifft, begann es erfreulich mit einem besonderen Geburtstag eines seiner Schriftleiter: Prof. Dr. med. Alex Kapp (• Abb. 1) wurde am 28. Februar 65 Jahre, was an anderer Stelle der Zeitschriften der Springer NatureGruppe bereits gewürdigt wurde [1].

\section{Verdienste für die Zeitschrift Der Hautarzt}

Es ist aber uns ein besonderes Anliegen, hier auch die besonderen Verdienste von Prof. Kapp für diese Zeitschrift hervorzuheben. Er ist seit 2002 Schriftleiter der Zeitschrift Der Hautarzt und koordinierte von 2002 bis Ende 2010 das Peer-review-Verfahren für frei eingereichte Übersichten und Beiträge der Rubrik „Wie lautet Ihre Diagnose?“, von 2011 bis Ende 2019 die Begutachtung von Originalarbeiten und Kasuistiken. Jeder, der schon einmal solche Begutachtungs-

Hans F. Merk' · Jean Krutmann ${ }^{2}$

${ }^{1}$ Klinik für Dermatologie und Allergologie, RWTH Aachen University, Aachen, Deutschland

${ }^{2}$ IUF - Leibniz-Institut für Umweltmedizinische Forschung, Düsseldorf, Deutschland

\title{
Laudatio zum 65. Geburtstag von Herrn Prof. Dr. Alexander Kapp
}

verfahren organisiert hat, weiß um den damit verbundenen Aufwand. Darüber hinaus wurden auf Anregung von Prof. Kapp mit Ausgabe 1/2003 die Leitthemenhefte eingeführt, die wesentlich zur Profilschärfung dieser Zeitschrift beigetragen haben und neben den CME-Beiträgen Kernstück des Heftes geworden sind.

\section{Beruflicher Werdegang}

Humanmedizin studierte Alexander Kapp als Kurpfälzer natürlich an der Ruprecht-Karls-Universität Heidelberg, und sein bleibender wissenschaftlicher Schwerpunkt zeigte sich schon bei der Promotion „T-Lymphozytenfunktion bei Neurodermitis atopica“. Er erweiterte seine Forschungsaktivitäten im Institut für Immunologie in Heidelberg und dem Max Planck Institut für Immunbiologie und Epigenetik in Freiburg, wo er in der inzwischen von seinem Doktorvater - Prof. Erwin Schöpf - geleiteten Hautklinik nach der Habilitation „Zur Aktivierung des oxidativen Stoffwechsels polymorphkerniger neutrophiler Granulozyten durch Zytokine aus mononukleären Zellen und Epidermalzellen“ den Funktionsbereich „Allergologie und Immundiagnostik“ etablierte.

In seiner Freiburger Zeit offenbarte sich rasch das beeindruckende Organisationstalent, über das Alexander Kapp verfügt. Leitender Oberarzt der UniversitätsHautklinik, Leiter des oben genannten klinischen und wissenschaftlichen Funktionsbereichs und zudem, je nach Bedarf, „Baubeauftragter“, Kongressorganisator und vieles mehr. Nicht zuletzt war es Alexander Kapp, der wesentlichen Anteil an der Initiierung und dem syste- matischen Aufbau des Forschungsprogramms der Freiburger Hautklinik hatte und beispielsweise ausschlaggebend war für die erfolgreiche Rekrutierung eines der beiden Unterzeichnenden als Assistenzarzt an diese Klinik. Letzterer hat sich dann auch in Freiburg sehr wohlgefühlt, und dies war nicht nur der kulinarisch schwer zu toppenden Umgebung, sondern auch dem Humor seines damaligen Oberarztes Alexander Kapp geschuldet, der so manches „bon mot“ prägte.

Im Jahr 1994 wurde Alexander Kapp Lehrstuhlinhaber und ärztlicher Direktor der Klinik für Dermatologie, Allergologie und Venerologie der Medizinischen Hochschule Hannover. Sein wissenschaftlicher und klinischer Schwerpunkt blieb natürlich die Erforschung pathophysiologischer Grundlagen entzündlicher Hauterkrankungen, und er machte diese Klinik rasch $\mathrm{zu}$ einem Leuchtturm dermatologischer Allergologie und klinischer Immunologie. Wichtige Themenfelder dieses Gebietes verbanden sich rasch mit Oberärzten seiner Klinik wie Urtikaria und Intoleranzreaktionen mit Frau Prof. Wedi, atopische Dermatitis mit Herrn Prof. Werfel, Juckreiz und bullöse Autoimmunerkrankungen mit Frau Prof. Raap, die inzwischen Ordinaria und Klinikdirektorin der Univ.-Hautklinik Oldenburg ist. Wesentliche Beiträge zur Fortentwicklung der spezifischen Hyposensibilisierung bei Insektengiftallergien und zur Anwendung der sublingualen Hyposensibilisierung folgten ebenfalls, die Alexander Kapp als Prüfarzt internationaler Multicenterstudien begleitete. In seine Zeit als Klinikdirektor fiel auch die Aufgabe, den Umzug der Klinik von der Hautklinik Linden auf den Campus der Medizinischen Hochschule zu or- 
ganisieren. Es gibt Beispiele namhafter Ordinarien, die diese nicht immer sehr erwünschte Ablenkung von eigentlichen wissenschaftlichen Themen mit einem Sabbatical verbanden und nach von Oberärzten organisiertem Umzug sich mit an anderer Stelle erarbeiteten wissenschaftlichen Beiträgen zurückmeldeten. Nicht so Alexander Kapp, er übernahm auch diese Aufgabe und machte sie zu seinem Anliegen neben allen anderen Interessen und Verpflichtungen, was zur Etablierung einer modernen Hautklinik einschließlich DIN-zertifizierten Qualitätsmanagementsystems führte. Alle diese Leistungen von Alexander Kapp führten zu zahlreichen Auszeichnungen, zuletzt 2013 der Erich-Fuchs-Preis des Ärzteverbandes Deutscher Allergologen (AeDA).

\section{Persönliches}

Als Kurpfälzer, der auch mehrere Jahre in Baden verbracht hat, zwingen freilich Hannover und Umgebung im önologischen und kulinarischen Bereich $\mathrm{zu}$ einigen Kompromissen. Wir konnten aber dankbar miterleben, dass Alexander Kapp das bei Kongressen in anderen Regionen und Ausflügen in die Kurpfälzer Heimat gut ausgleichen kann. Besonders verpflichtet ist Alexander Kapp auch seiner Familie. Seine Ehefrau Gabriele, die nicht nur zu Beginn seiner Karriere bedeutende elektronenmikroskopische Studien beigetragen hatte, begleitet ihn bei seinen weiteren Interessen wie Tanzsport und Opernbesuche, die Tochter ist inzwischen stolze Wirtschaftsanwältin, und sein Sohn hat ebenfalls den Weg zur Dermatologie gefunden.

Bei all diesen Aktivitäten freuen wir uns, über das besondere Jahr 2020 hinaus auf die weitere Zusammenarbeit mit Alexander Kapp, nicht zuletzt bei der Herausgabe dieser Zeitschrift.

Hans Merk

Jean Krutmann

\section{Korrespondenzadresse}

\section{Prof. Dr. Hans F. Merk}

Klinik für Dermatologie und Allergologie, RWTH Aachen University

Pauwelsstr. 30, 52074 Aachen, Deutschland

hans.merk@post.rwth-aachen.de

\section{Literatur}

1. Czech W, Wedi B, Werfel $T$ (2020) Immer im Einsatz für Dermatologie und Allergologie. Allergo J 29:68-69

\section{Leitthemenübersicht Der Hautarzt 2020/2021}

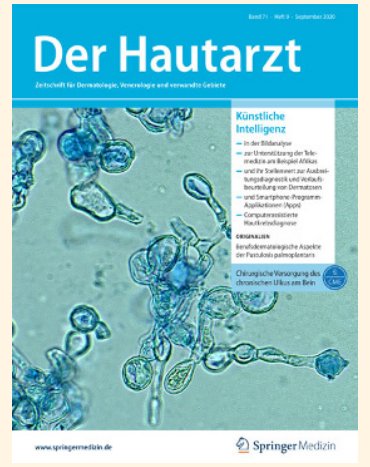

Der Hautarzt bietet Ihnen umfassende und aktuelle Beiträge zu interessanten Themenschwerpunkten aus allen Bereichen der Dermatologie einschließlich Allergologie, Venerologie und ihren verwandten Gebieten.

\section{Halbjahr 2020 \\ - 7/20 Pruritus \\ - 8/20 Epitheliale Tumoren \\ - 9/20 Künstliche Intelligenz \\ - 10/20 Dermatoendokrinologie \\ - 11/20 Wundheilung \\ - 12/20 Pigmentstörungen}

\section{Halbjahr 2021 \\ - 1/21 Photobiologie \\ - 2/21 Impfungen \\ - 3/21 Kinderdermatologie \\ - 4/21 Paraneoplastische Syndrome \\ - 5/21 Ästhetische Dermatologie \\ - 6/21 Berufsdermatologie \\ (Änderungen vorbehalten)}

Einzelne Ausgaben können Sie direkt bei unserem Kundenservice zum Preis von je EUR 44,- (2021: EUR 45,-) zzgl. Versandkosten beziehen. So erreichen Sie unseren Kundenservice:

Springer Nature Customer Service Center $\mathrm{GmbH}$

Kundenservice Zeitschriften

Tiergartenstr. 15, 69121 Heidelberg

Tel.: +496221345-4303

leserservice@springernature.com

www.DerHautarzt.de 